\title{
Electromagnetic-guided versus endoscopic placement of post-pyloric feeding tubes: a systematic review and meta-analysis of randomised controlled trials
}

Yaping $\mathrm{Wei}^{\dagger}$, Zheng $\mathrm{Jin}^{\dagger}$, Ying Zhu and Wei Hu${ }^{*}$

\begin{abstract}
Background: Current evidence supporting the utility of electromagnetic (EM)-guided method as the preferred technique for post-pyloric feeding tube placement is limited. We conducted a meta-analysis to compare the performance of EM-guided versus endoscopic placement.

Methods: We searched several databases for all randomised controlled trials evaluating the EM-guided vs. endoscopic placement of post-pyloric feeding tubes up to 28 July 2020. Primary outcome was procedure success rate. Secondary outcomes included reinsertion rate, number of attempts, placement-related complications, tuberelated complications, insertion time, total procedure time, patient discomfort, recommendation scores, length of hospital stay, mortality, and total costs.
\end{abstract}

Results: Four trials involving 536 patients were qualified for the final analysis. There was no difference between the two groups in procedure success rate (RR 0.97; 95\% Cl 0.91-1.03), reinsertion rate (RR 0.84; 95\% Cl 0.59-1.20), number of attempts (WMD - 0.23; 95\% Cl - 0.99-0.53), placement-related complications (RR 0.78; 95\% Cl 0.41-1.49), tube-related complications (RR 1.08; 95\% Cl 0.82-1.44), total procedure time (WMD - 18.09 min; 95\% Cl - 38.662.47), length of hospital stay (WMD 1.57 days; $95 \%$ Cl - 0.33-3.47), ICU mortality (RR 0.80; 95\% Cl 0.50-1.29), inhospital mortality (RR 0.87; 95\% Cl 0.59-1.28), and total costs (SMD - 1.80; 95\% Cl - 3.96-0.36). The EM group was associated with longer insertion time (WMD 4.3 min; 95\% Cl 0.2-8.39), higher patient discomfort level (WMD 1.28; 95\% Cl 0.46-2.1), and higher recommendation scores (WMD 1.67; 95\% Cl 0.24-3.10).

Conclusions: No significant difference was found between the two groups in efficacy, safety, and costs. Further studies are needed to confirm our findings.

Systematic review registration: PROSPERO (CRD42020172427)

Keywords: Electromagnetic, Endoscopy, Post-pyloric feeding tube, Enteral nutrition

\footnotetext{
* Correspondence: jzercp@126.com

${ }^{\dagger}$ Yaping Wei and Zheng Jin contributed equally to this work.

Affiliated Hangzhou First People's Hospital, Zhejiang University School of Medicine, Hangzhou, China
}

(c) The Author(s). 2020 Open Access This article is licensed under a Creative Commons Attribution 4.0 International License, which permits use, sharing, adaptation, distribution and reproduction in any medium or format, as long as you give appropriate credit to the original author(s) and the source, provide a link to the Creative Commons licence, and indicate if changes were made. The images or other third party material in this article are included in the article's Creative Commons licence, unless indicated otherwise in a credit line to the material. If material is not included in the article's Creative Commons licence and your intended use is not permitted by statutory regulation or exceeds the permitted use, you will need to obtain permission directly from the copyright holder. To view a copy of this licence, visit http://creativecommons.org/licenses/by/4.0/. The Creative Commons Public Domain Dedication waiver (http://creativecommons.org/publicdomain/zero/1.0/) applies to the data made available in this article, unless otherwise stated in a credit line to the data. 


\section{Background}

Malnutrition and inability to eat are conditions often encountered in inpatients. For such patients, enteral nutrition is considered to be superior to parenteral nutrition since it reduces complications, improves patient outcome, and is cheaper [1,2]. It is common practice to place a post-pyloric feeding tube for enteral nutrition in patients who are intolerant of intragastric nutrition [3]. Endoscopic technique is typically used, but may require patient transportation between wards, pre-procedural fasting, and radiological confirmation of the tube's position. Since first reported by Phang et al. in 2006 [4], electromagnetic (EM)-guided technique has been increasingly used for post-pyloric feeding tube placement. It has been suggested to be convenient and lead to a significant cost reduction. With increasing availability and familiarity with this technique, several randomised controlled trials (RCTs) [5-8] have compared EM-guided versus endoscopic (ENDO) technique. These RCTs were limited because of small sample sizes. We therefore conducted a meta-analysis to compare the performance between EM and ENDO.

\section{Methods}

This meta-analysis follows the Preferred Reporting Items for Systematic Reviews and Meta-Analysis (PRISMA) statement [9] and was registered on PROSPERO (CRD42020172427).

\section{Search strategy}

Two investigators (Y-W and Y-Z) independently searched MEDLINE, EMBASE, the Cochrane Library, and Google Scholar for all entries through 28 July 2020 using the following search terms: "Cortrak", "electromagnetic", "endoscopic", "nasoenteral, or post-pyloric", and "tube(s), feeding, or nutrition" (see Supplemental Digital Content 1, for exemplar PubMed search). Then, they compared their lists of potentially eligible titles and abstracts and achieved a consensus on full review.

\section{Study selection criteria}

The following criteria were used to select studies for inclusion: (i) studies directly compared EM-guided versus endoscopic placement of post-pyloric feeding tubes; (ii) RCTs; and (iii) were English language articles. All retrospective studies, non-controlled studies, reviews, case series, abstracts, editorials, letters to editor, animal studies, duplicate studies, and studies without data on any of the primary or secondary outcomes were excluded.

\section{Study selection and data extraction}

Decisions about study inclusion and exclusion were made independently by two investigators ( $\mathrm{Y}-\mathrm{W}$ and $\mathrm{W}$ $\mathrm{H})$. Two investigators (Z-J and $\mathrm{W}-\mathrm{H})$ extracted data independently from each study. We collected author, year of publication, country of origin, number of centres, participating operators, patient demographics, indications for enteral nutrition, and study outcomes. Any discrepancies were resolved by mutual discussion.

\section{Outcome measures}

Our primary outcome was procedure success rate (defined as the percentage of successful tube placement in the desired location as determined). Secondary outcomes included reinsertion rate (defined as the percentage of patients undergoing reinsertion after an unsuccessful primary procedure or dislodgement/blockage of the tube), number of attempt, placement-related complications (e.g. epistaxis, gastrointestinal tract blood, and abdominal pain), tube-related complications (e.g. dislodgement, blockage, and aspiration), insertion time (defined as the time interval from insertion of the tube until fixation of the tube to the nostrils), total procedure time (including time for preparation and recovery), patient discomfort (recorded with scores in the range 0 [no complaints] to 10 [maximum complaints]), patient recommendation (recorded with scores in the range 0 [not recommended] to 10 [highly recommended]), length of hospital stay, intensive care unit (ICU) mortality, in-hospital mortality, and total costs (including the costs for tube placement procedure, complications, and therapeutic interventions).

\section{Validity assessment}

Risk of bias assessment of RCTs was performed independently by two investigators ( $\mathrm{Y}-\mathrm{W}$ and $\mathrm{Z}-\mathrm{J}$ ) using the Cochrane Collaboration's tool [10]. Any disagreements were resolved by consensus after a mutual discussion. We also assessed the confidence in the estimates derived using the Grading of Recommendations Assessment, Development, and Evaluation (GRADE) approach. As all the studies included are RCTs, using the GRADE system they are considered of high quality and are downgraded to levels of moderate, low, or very low quality based on the risk of bias, indirectness, imprecision, inconsistency, and publication bias [11].

\section{Statistical analysis}

Risk ratios (RRs) were calculated for categorical variables. Studies with no events in both arms were excluded from the meta-analysis of RRs [12]. Standard mean differences (SMDs) were calculated for continuous variables including total costs based on different monetary units. Weighted mean differences (WMDs) were calculated for the rest of continuous variables. In cases of missing data, especially for continuous variables, we estimated the mean and standard deviation from the sample size, median, range, and/or interquartile range, if available, according to the approximation method previously validated [13]. Owning to variation in study protocols and study populations, a random effects 
model for all analyses was used [12]. Heterogeneity among studies was assessed by calculating the $I^{2}$ statistics and tested using the Cochrane Q-test [14]. Subgroup analyses were performed for all outcomes based on the following: (a) study setting (single- vs. multicentre), (b) geographical location (Asia vs. Europe), (c) the BMI level of patients with the validated method (ENDO group) $(<25$ vs. $>25)$, (d) prior altered upper gastrointestinal anatomy (with vs. without), (e) patient population (critically ill vs. non-critically ill patients). For those subgroups with only one study included, subgroup analyses were not performed. We had planned that if enough studies $(\geq 10)$ were included in the analysis of primary outcome, we would construct a funnel plot to evaluate publication bias [12]; otherwise, Egger's test was applied [15]. Statistical analyses were performed using Review Manager 5.3 (The Cochrane Collaboration, The Nordic Cochrane Centre, Copenhagen, Denmark).

\section{Results}

\section{Search strategy yield and study characteristics}

The search identified 176 potentially relevant studies, 15 of which were examined in detail and 4 RCTs of which were included in the final analysis [15] (Fig. 1). A total of 536 patients were included, of which 287 were in the EM group and 249 in the ENDO group. Mean age was 57.1 years with a range of 51.5 to 64.6 years. The sex distribution was $53.3 \%$ male patients, with a range of 51.3 to $63.6 \%$. Two studies were conducted in Netherlands [6, 7], one in Austria [5], and one in China [8]. Two of the studies [8] were of multicentre design ( 3 or 5 recruiting sites). Two studies $[5,8]$ exclusively evaluated critical ill patients, one [6] evaluated patients from gastrointestinal surgical wards, and the remaining one [7] included outpatients, ward patients, and critically ill patients. In the ENDO group, tube placement was performed by a gastroenterologist assisted by one or two nurses. For EM group, tube placement was performed by one nurse in two studies [6,7], by a nutritional support team in one study [8], and the remaining one study [5] did not specify this issue. One study [6] included patients with prior altered upper gastrointestinal anatomy and the remaining three studies did not. For EM technique, pre-procedural fasting was required in one study [7], not required in one study [6], and the remaining two studies $[5,8]$ did not specify this issue. Conscious sedation in the EM group was not required in two studies

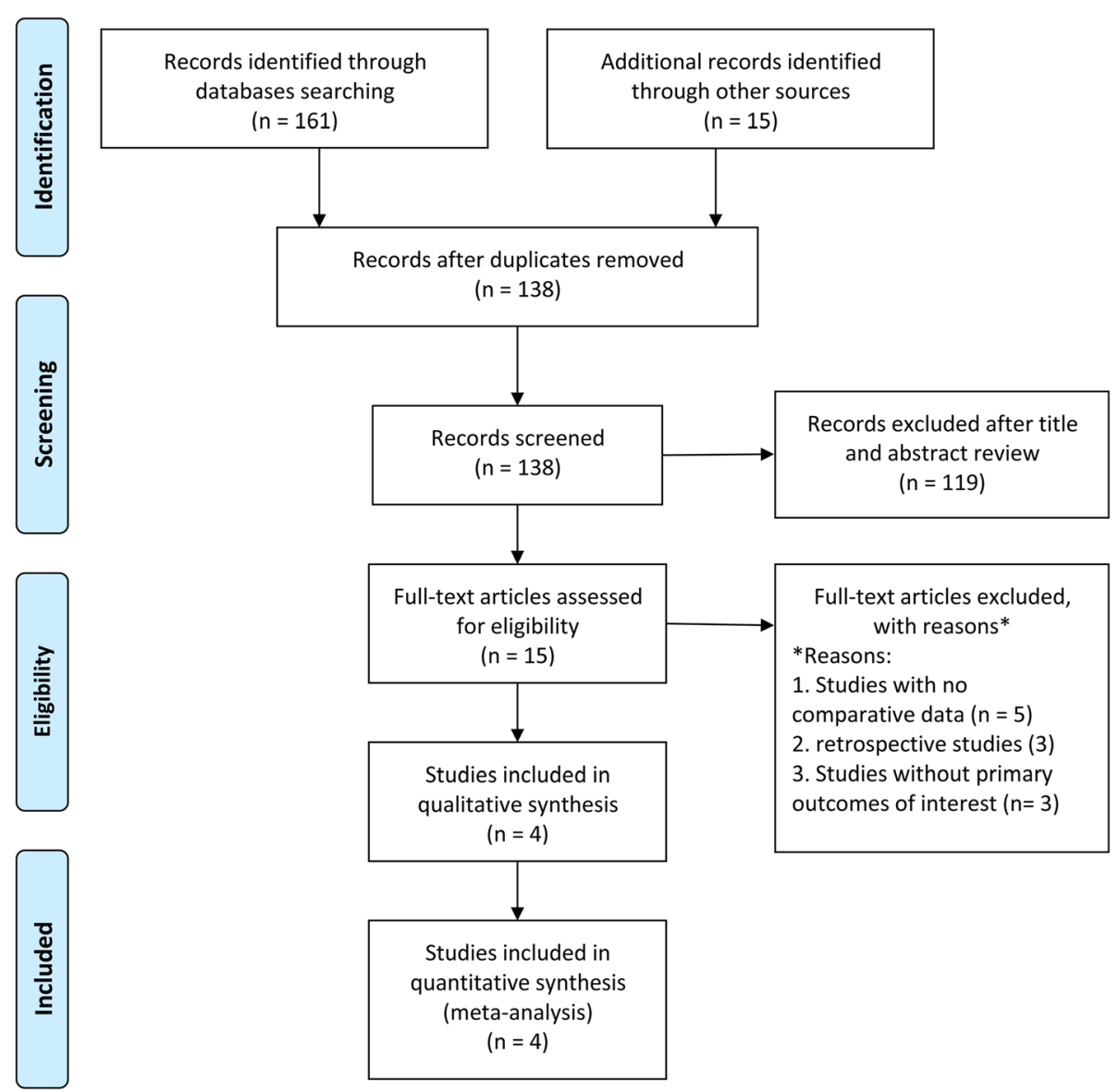

Fig. 1 The PRISMA flow diagram of selected studies 
$[6,8]$, but was used if indicated in the remaining two studies $[5,7]$. For the ENDO group, conscious sedation was used in a large portion of patients. Details of the characteristics of included studies are given in Table 1.

\section{Quality assessment}

All of the included studies had a high risk of performance bias. This could not be avoided because the operators and patients could not be blinded to the method of examination. There was unclear risk of detection bias in all studies, as blinding of outcome assessment could only be done for subjective outcomes such as patient discomfort and patient recommendation. One study [5] had an unclear risk of selection bias owing to no reported concealment of allocations. Assessment of risk of bias is shown in Supplemental Digital Content 2.

\section{Primary outcome: procedure success rate}

Procedure success rate was reported in all included studies (Table 2). Pooled rate for EM and ENDO was 82.6\% and $83.1 \%$, respectively. No statistical difference was observed (RR 0.97; 95\% confidence interval [95\% CI] 0.911.03; $I^{2}=0 \%$; GRADE = moderate) (Fig. 2a). We did not employ funnel plot to access for publication bias as fewer than 10 studies were included. There was no evidence of publication bias by Egger' $\mathrm{s}$ test for the primary outcome $(P=0.18)$.

\section{Secondary outcomes}

Two studies [6, 7] with 281 patients were included to evaluate reinsertion rate. Of 148 patients undergoing EM-guided placement, reinsertion occurred in 41 patients (27.7\%). As for ENDO, reinsertion occurred in 44 patients (33.1\%). There was no significant difference between two groups (RR 0.84; 95\% CI $0.59-1.20 ; I^{2}=0 \%$; GRADE $=$ moderate) (Fig. 2b). Number of attempts was reported in 2 studies $[5,8]$. No significant difference was found between two groups (1.2 vs. 1.5; WMD - 0.23; 95\% CI - 0.99-0.53; $I^{2}=94 \%$; GRADE = low) (Fig. 2c).

All studies evaluated placement-related complications. In the EM group, placement-related complications occurred in 18 patients (6.3\%), which mainly contained 10 epistaxis (3.5\%), 1 gastrointestinal tract blood (0.3\%), 1 hypoxia (0.3\%), 1 atrial fibrillation $(0.3 \%)$, and 1 abdominal pain (0.3\%). As for ENDO, placement-related complications occurred in 18 patients (7.2\%), which mainly contained 12 epistaxis (4.8\%), 1 gastrointestinal tract blood $(0.4 \%)$, and 4 hypoxias (1.6\%). No significant difference was found between the two groups (RR $0.78 ; 95 \%$ CI 0.41-1.49; $I^{2}=0 \%$; GRADE = moderate) (Fig. 3a). Two studies $[6,8]$ with 315 patients reported tube-related complications. In the EM group, tube-related complications occurred in 57 patients (35.4\%), which mainly contained 45 dislodgements (28.0\%), and 13 blockages (8.1\%). As for
ENDO, tube-related complications occurred in 50 patients (32.5\%), which mainly contained 38 dislodgements (24.7\%), 7 blockages (4.5\%), and 3 aspirations (1.9\%). No significant difference was found in total tube-related complications (RR 1.08; 95\% CI 0.82-1.44; $I^{2}=0 \%$; GRADE = moderate) (Fig. 3b). No procedure-related mortality was reported among studies.

All included studies evaluated insertion time. EM was associated with longer insertion time than ENDO (14.8 min vs. $10.8 \mathrm{~min}$; WMD $4.3 \mathrm{~min}$; $95 \%$ CI $0.2-8.39 ; I^{2}=$ $89 \%$; GRADE = low) (Fig. 4a). Total procedure time was reported in 2 studies $[6,8](n=315)$. No significant difference was found between two groups (24.5 min vs. 43 min; WMD - $18.09 \mathrm{~min} ; 95 \% \mathrm{CI}-38.66-2.47 ; I^{2}=96 \%$; GRADE = low) (Fig. 4b).

Patient-assessed discomfort was reported in 2 studies [6, 8] $(n=315)$. EM showed higher discomfort level than ENDO (WMD 1.28; 95\% CI 0.46-2.1; $I^{2}=47 \%$; GRADE = moderate) (Fig. 4c). Patient recommendation was reported in 3 studies [6-8] $(n=470)$. The EM group received higher recommendation scores than the ENDO group (WMD 1.67; 95\% CI 0.24-3.10; $I^{2}=83 \%$; GRADE = low) (Fig. 4d).

Length of hospital stay was reported in 2 studies [6-8] $(n=315)$. No significant difference was found between the 2 groups (14.8 days vs. 13.2 days; WMD 1.57 days; $95 \%$ CI - 0.33-3.47; $I^{2}=0 \%$; GRADE = moderate) (Fig. 5a). Mortality was reported in 3 studies $[5,6,8](n=381)$. There was no difference between the two groups in ICU mortality (RR 0.80; 95\% CI 0.50-1.29; $I^{2}=0 \%$; GRADE = moderate) (Fig. 5b) and in-hospital mortality (RR 0.87; 95\% CI 0.59-1.28; $I^{2}=0 \%$ ) (Fig. 5c).

Total costs were provided in 3 studies [6-8] $(n=470)$. Currency units were variably used euro and dollar. No significant difference was found between the two groups $\left(\mathrm{SMD}-1.80\right.$; 95\% CI - 3.96-0.36; $I^{2}=99 \%$; GRADE = low) (Fig. 5d). Table 3 shows the quality of evidence using the GRADE assessment tool which is detailed for each of the outcomes.

\section{Subgroup analyses}

Subgroup analyses were performed for all outcomes (Supplemental Digital Content 3). EM compared with ENDO was associated with lower total cost when only evaluating multicentre RCTs (SMD - 0.29; 95\% CI 0.51 to $-0.06 ; I^{2}=0 \%$ ). Other subgroup analysis results were accordant to the main analyses. Heterogeneity was reduced when subgroup analyses for total costs and insertion time was performed using level of BMI $<25$ as a modifier. The other heterogeneity could not be explained by these variables with the available data.

\section{Discussion}

The conventional methods for the placement of postpyloric feeding tubes include blind, fluoroscopic, and 


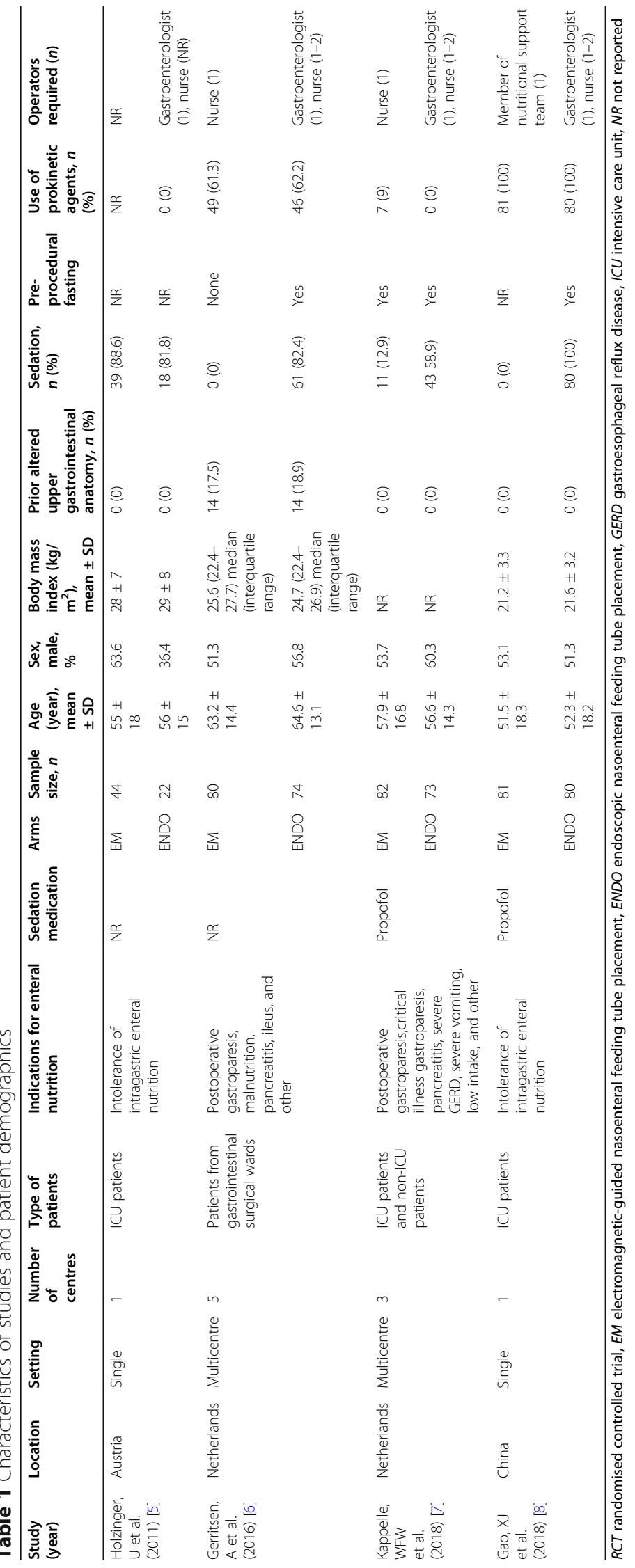




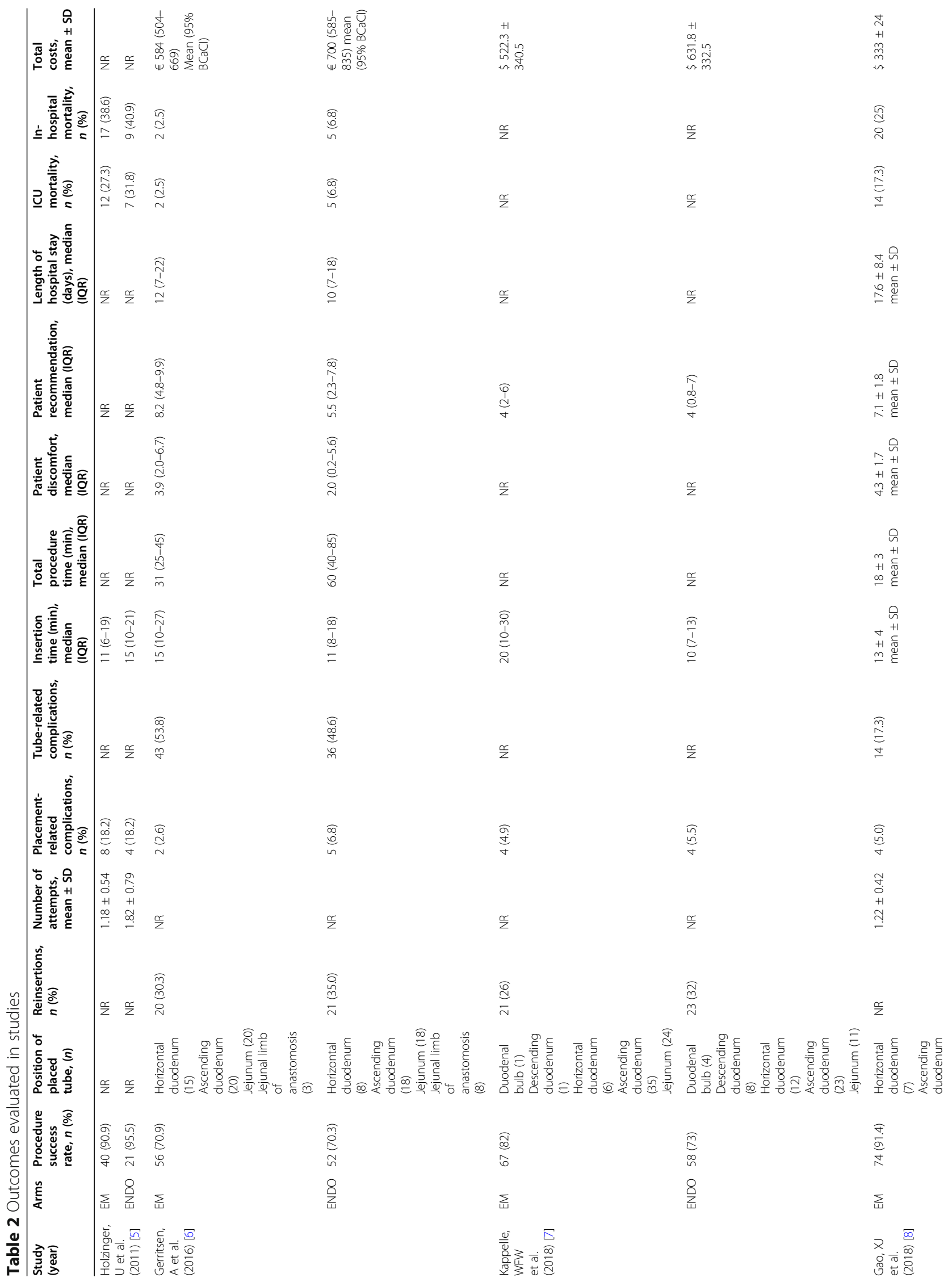




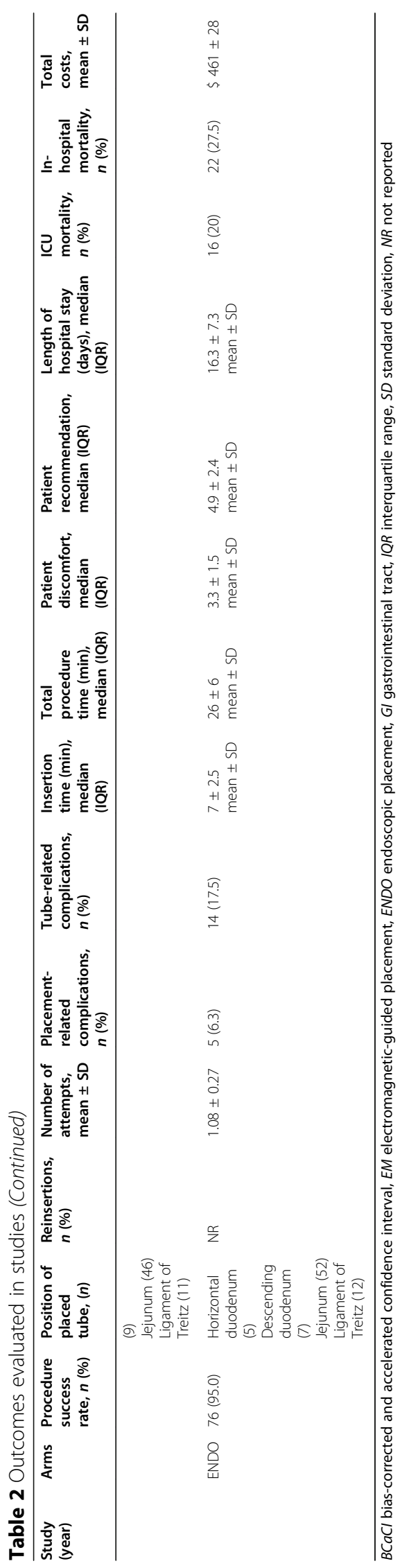




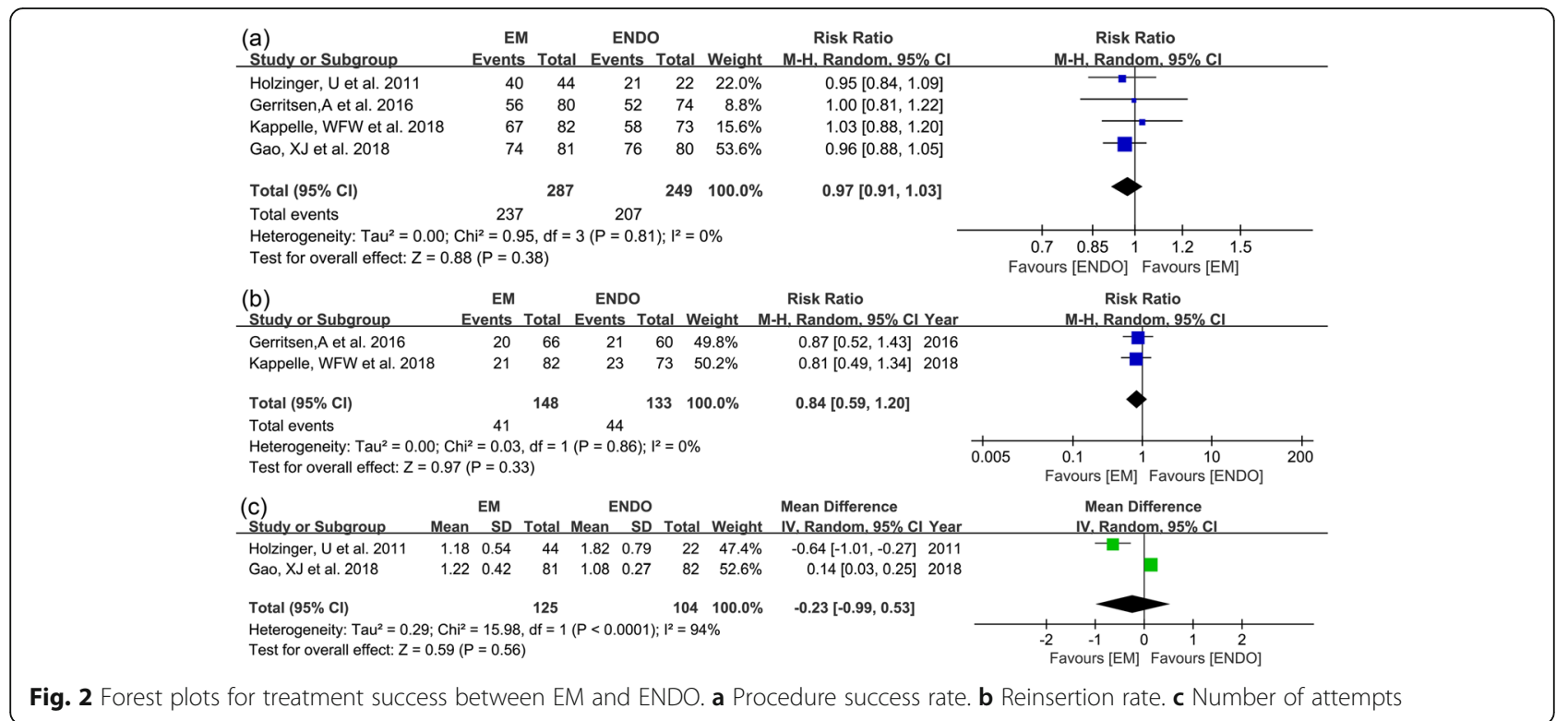

endoscopic methods [16, 17]. The gold standard is the endoscopic technique, which has success rates above 90\% [18, 19]. Bedside EM-guided tube placement can be performed in recent years [20]. This has several potential advantages compared with endoscopic placement because only one trained nurse and less equipment is needed [21, 22]. EM and ENDO techniques have been compared in only one systematic review until now [23]. That review by Gerritsen et al. involving only one relevant RCT (66 patients) concluded that the efficacy and safety of the two techniques did not differ significantly, but EM offered advantages in logistics. In view of 3 new RCTs published, we have attempted to pool the evidence to further evaluate the performance of EM vs. ENDO.

Regarding treatment success, our meta-analysis did not demonstrated difference in procedure success rate, reinsertion rate, and number of attempts. These results were robust across the subgroup analyses. Our procedure success rates of the two groups $(82.6 \%$ vs. $83.1 \%)$ were lower than those reported in the previous systematic review ( $85 \%$ vs. $89 \%$ ), and our reinsertion rates ( $27.7 \%$ vs. $33.1 \%)$ were higher than theirs $(21 \%$ vs. $16 \%)$. The reasonable explanation may be that our review included many gastrointestinal disease patients, with an altered upper gastrointestinal anatomy, which may hamper tube placement.

With respect to safety, our meta-analysis did not provide evidence that difference existed in complication rates, length of hospital stay, ICU mortality, and inhospital mortality. Subgroup analysis results were consistent with main analyses in these outcomes. Average placement- and tube-related complication rates for EM that have been reported in previous review was $0.4 \%$ and $15 \%$, our finding $(6.3 \%$ and $35.4 \%)$ was significantly 


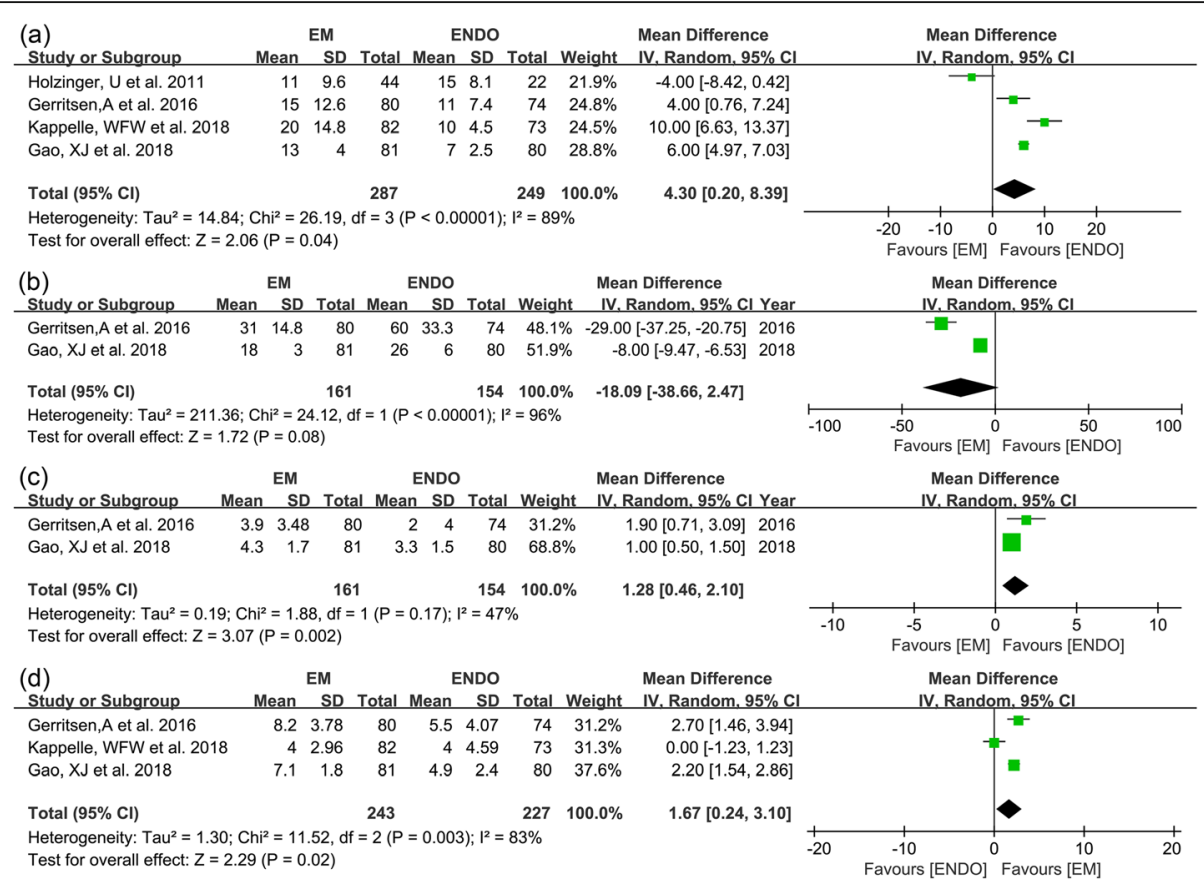

Fig. 4 Forest plots for a insertion time, $\mathbf{b}$ total procedure time, $\mathbf{c}$ patient discomfort, and $\mathbf{d}$ patient recommendation between EM and ENDO

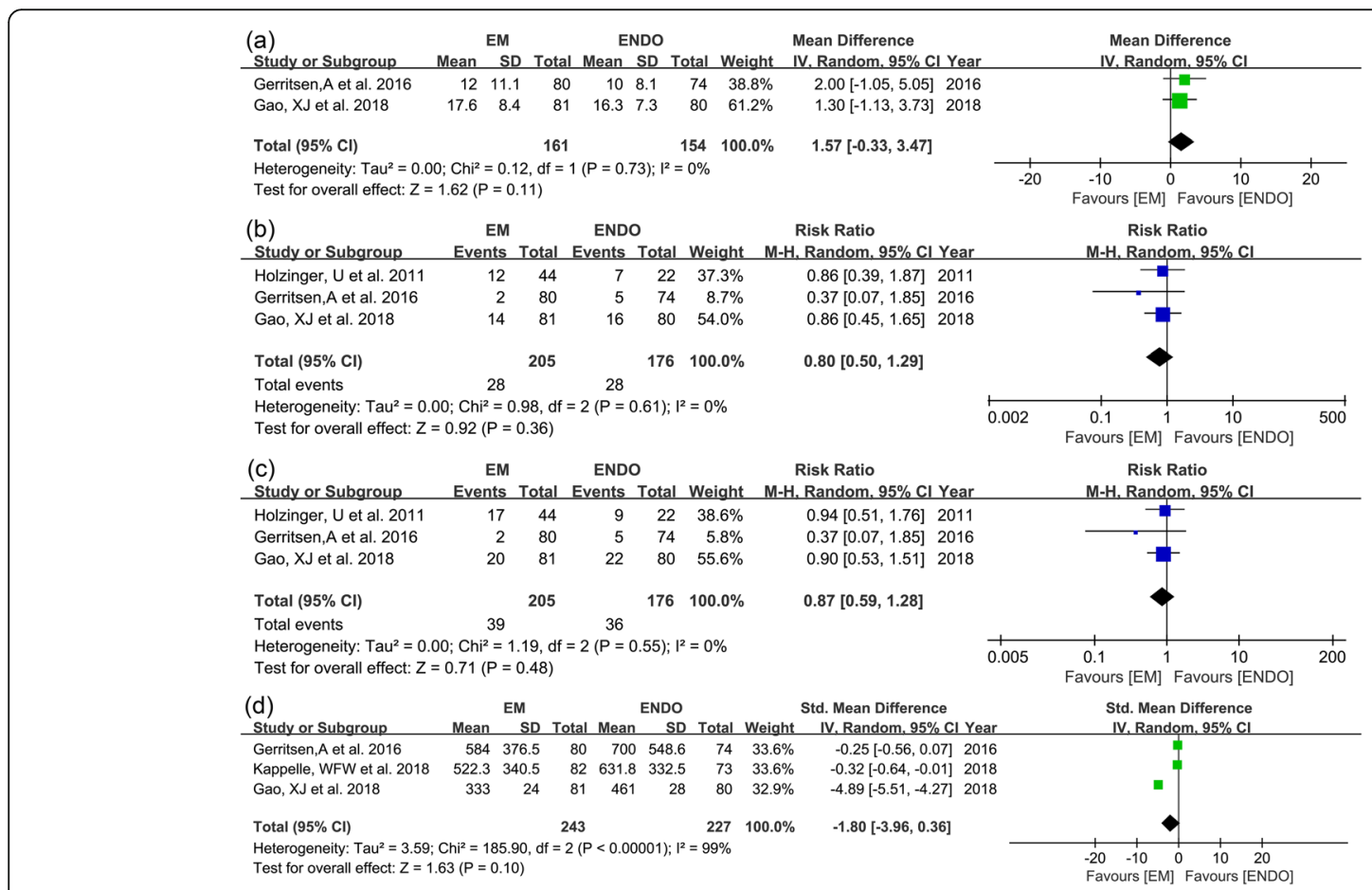

Fig. 5 Forest plots for a length of hospital stay, $\mathbf{b}$ ICU mortality, $\mathbf{c}$ in-hospital mortality, and $\mathbf{d}$ total costs of the feeding tube placement between EM and ENDO 


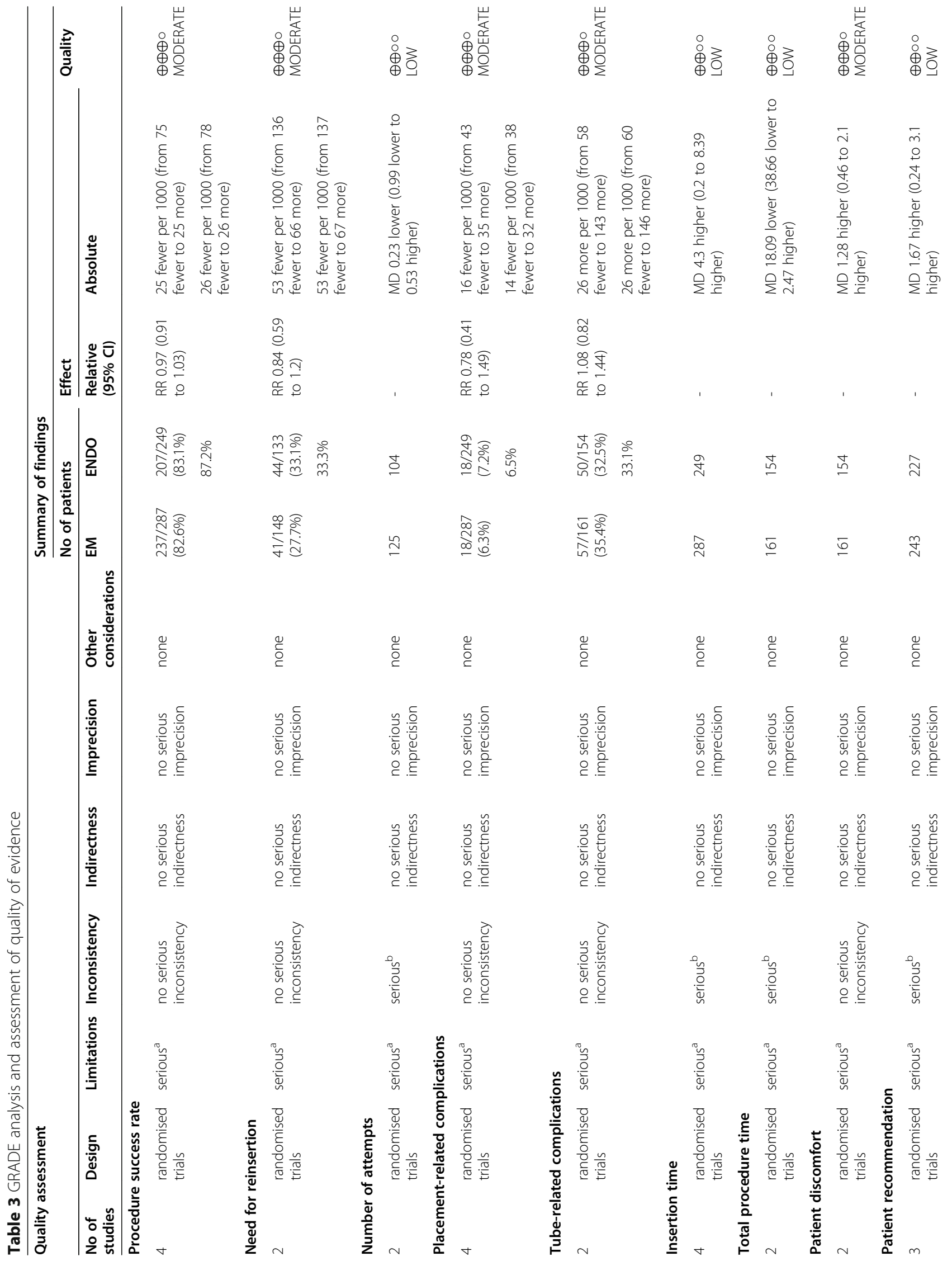




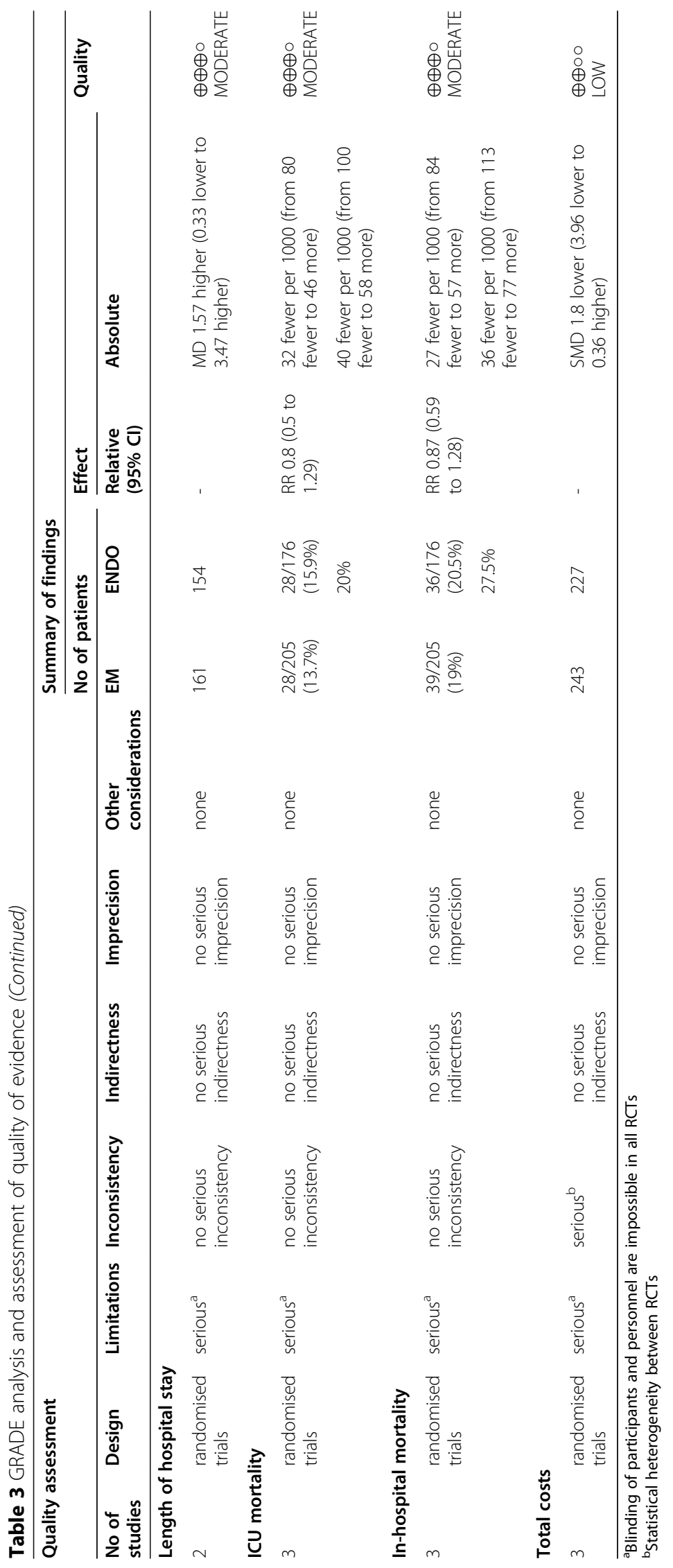


higher than theirs. The most common complications in our review were epistaxis and dislodgment/blockage of the tube. However, undetected statistical difference in complications may be attributed to low incidence and insufficient sample size. More large-sample RCTs are required to investigate these outcomes in greater detail. It is worth noting that, unlike EM, sedation is often required during endoscopic technique. Sedation is associated with a small risk of cardiopulmonary adverse events, e.g. hypoxia [24]. This could account for the difference in hypoxia incidence between the two techniques ( $0.3 \%$ vs. $1.6 \%)$.

Although EM was more time consuming for insertion, the fact that EM technique does not require sedation and patient transportation resulted in comparable total time compared with endoscopy. Another advantage of EM is that in case of partial migration of the tube, repositioning of the tube can be done by reinserting the stylet through the tube at the bedside, whereas repositioning by endoscopy would require removal of the tube and repetition of the entire procedure (including sedation). Regarding patient-reported outcomes, patients reported more discomfort during EM-guided placement than during endoscopy. This finding is probably related to the large differences in the use of conscious sedation between the two groups. On the other hand, recommendation scores were significantly higher in the EM group, presumably because the discomfort is not bothersome enough to advise others not to undergo the EM procedure. This supports the hypothesis that EM is a more patientfriendly approach.

Finally, as for total costs, we found absence of evidence for difference between the two groups. The reduction in the use of hospital resources (e.g. personnel, patient transportation) and the absence of the need for sedation and radiographic confirmation can lead to a reduction in costs. Therefore, the cost difference between EM and ENDO we found may be an underestimation of the true cost difference. This could also be evidenced in subgroup analyses: lower total cost with EM was noted, when only evaluating multicentre RCTs. More welldesigned multicentre RCTs are therefore warranted.

Our meta-analysis has several strengths. First, we conducted rigorous search of the RCTs and added 3 additional studies that the previous systematic review did not include. Second, comprehensive subgroup analyses were performed. Last, we assessed multiple clinically relevant outcomes. Because critically ill and non-critically ill patients were both included, the results of our review are probably generalizable to the overall hospital population. However, limitations are present and are as follows. The number of included studies is relatively small. Variation among studies was observed in study design, introducing heterogeneity.
Data on patients with prior altered upper gastrointestinal anatomy was not enough for subgroup analyses.

\section{Conclusions}

Based on the currently available literature, our metaanalysis does not demonstrate the difference between EM and ENDO in safety, efficacy, and total cost. Further studies are needed to confirm our findings.

\section{Supplementary Information}

The online version contains supplementary material available at https://doi. org/10.1186/s40560-020-00506-8.

Additional file 1: Supplemental Digital Content 1. PubMed search strategy. Supplemental Digital Content 2. Assessment of risk of bias. Green denotes low risk of bias, red indicates high risk of bias, and yellow represents unclear risk of bias. Supplemental Digital Content 3.

Subgroup analyses (EM vs. ENDO).

\section{Abbreviations}

EM: Electromagnetic; ENDO: Endoscopic; PRISMA: the Preferred Reporting Items for Systematic Reviews and Meta-Analysis; RCT: Randomised clinical trial; RR: Risk ratio; SD: Standard deviation; SMD: Standard mean difference; WMD: Weighted mean difference

\section{Acknowledgements \\ None.}

\section{Authors' contributions}

Y-W contributed to the study design, acquisition of data, statistical analysis, and drafting of the manuscript; Z-J contributed to the statistical analysis and critical revision of the manuscript; $Y-Z$ contributed to the acquisition of data and critical revision of the manuscript; $\mathrm{W}-\mathrm{H}$ contributed to the study design and critical revision of the manuscript. The author(s) read and approved the final manuscript.

\section{Funding}

This study was supported by grant from Zhejiang Province Medical Health Science and Technology Project (CN) (2020KY702).

Availability of data and materials

All the data supporting the conclusions of this article are included within the article.

Ethics approval and consent to participate Not applicable.

Consent for publication

All authors have agreed to the publication of this manuscript.

Competing interests

The authors declare that they have no competing interests.

Received: 20 August 2020 Accepted: 10 November 2020

Published online: 10 December 2020

References

1. Jones NE, Heyland DK. Implementing nutrition guidelines in the critical care setting: a worthwhile and achievable goal? JAMA. 2008;300:2798-9.

2. Doig GS, Heighes PT, Simpson F, Sweetman EA, Davies AR. Early enteral nutrition, provided within $24 \mathrm{~h}$ of injury or intensive care unit admission, significantly reduces mortality in critically ill patients: a meta-analysis of randomised controlled trials. Intensive Care Med. 2009;35:2018-27.

3. Hsu CW, Sun SF, Lin SL, Kang SP, Chu KA, Lin CH, et al. Duodenal versus gastric feeding in medical intensive care unit patients: a prospective, randomized, clinical study. Crit Care Med. 2009;37:1866-72. 
4. Phang J, Marsh W, Prager R. Feeding tube placement with the aid of a new electromagnetic transmitter: Jpen-Parenter Enter. 2006;30:582.

5. Holzinger U, Brunner R, Miehsler W, Herkner H, Kitzberger R, Fuhrmann V, et al. Jejunal tube placement in critically ill patients: a prospective, randomized trial comparing the endoscopic technique with the electromagnetically visualized method. Crit Care Med. 2011;39:73-7.

6. Gerritsen A, de Rooij T, Dijkgraaf MG, Busch OR, Bergman JJ, Ubbink DT, et al. Electromagnetic-guided bedside placement of nasoenteral feeding tubes by nurses is non-inferior to endoscopic placement by gastroenterologists: a multicenter randomized controlled trial. Am J Gastroenterol. 2016;111:1123-32.

7. Kappelle W, Walter D, Stadhouders PH, Jebbink H, Vleggaar FP, van der Schaar PJ, et al. Electromagnetic-guided placement of nasoduodenal feeding tubes versus endoscopic placement: a randomized, multicenter trial. Gastrointest Endosc. 2018;87:110-8.

8. Gao X, Zhang L, Zhao J, Tian F, Sun H, Wang P, et al. Bedside electromagnetic-guided placement of nasoenteral feeding tubes among critically III patients: a single-centre randomized controlled trial. J Crit Care. 2018:48:216-21.

9. Moher D, Liberati A, Tetzlaff J, Altman DG. Preferred reporting items for systematic reviews and meta-analyses: the PRISMA statement. BMJ. 2009, 339:b2535.

10. Higgins JP, Altman DG, Gotzsche PC, Juni P, Moher D, Oxman AD, et al. The Cochrane Collaboration's tool for assessing risk of bias in randomised trials. BMJ. 2011;343:d5928.

11. Guyatt GH, Oxman AD, Vist GE, Kunz R, Falck-Ytter Y, Alonso-Coello P, et al. GRADE: an emerging consensus on rating quality of evidence and strength of recommendations. BMJ. 2008;336:924-6.

12. Higgins JP, Green S. Cochrane Handbook for Systematic Reviews of Interventions. In; 2011.

13. Luo D, Wan X, Liu J, Tong T. Optimally estimating the sample mean from the sample size, median, mid-range, and/or mid-quartile range. Stat Methods Med Res. 2016;27:1785-805.

14. Higgins JP, Thompson SG. Quantifying heterogeneity in a meta-analysis. Stat Med. 2002;21:1539-58.

15. Egger M, Davey SG, Schneider M, Minder C. Bias in meta-analysis detected by a simple, graphical test. BMJ. 1997;315:629-34.

16. Hillard AE, Waddell JJ, Metzler MH, McAlpin D. Fluoroscopically guided nasoenteric feeding tube placement versus bedside placement. South Med J. 1995;88:425-8.

17. Scott R, Bowling TE. Enteral tube feeding in adults. J R Coll Physicians Edinb. 2015;45:49-54

18. Byrne KR, Fang JC. Endoscopic placement of enteral feeding catheters. Curr Opin Gastroenterol. 2006;22:546-50

19. Wiggins TF, DeLegge MH. Evaluation of a new technique for endoscopic nasojejunal feeding-tube placement. Gastrointest Endosc. 2006;63:590-5.

20. McCutcheon KP, Whittet WL, Kirsten JL, Fuchs JL. Feeding tube insertion and placement confirmation using electromagnetic quidance: a team review. Jpen-Parenter Enter. 2018;42:247-54.

21. Kline AM, Sorce L, Sullivan C, Weishaar J, Steinhorn DM. Use of a noninvasive electromagnetic device to place transpyloric feeding tubes in critically ill children. Am J Crit Care. 2011;20:453-60.

22. Arjaans W, Ouwehand M, Bouma G, van der Meulen T, de van der Schueren M. Cortrak((R)) duodenal tube placements: a solution for more patients? A preliminary survey to the introduction of electromagnetic-guided placement of naso-duodenal feeding tubes. Clin Nutr ESPEN. 2019;29:133-6.

23. Gerritsen A, van der Poel MJ, de Rooij T, Molenaar IQ, Bergman JJ, Busch $\mathrm{OR}$, et al. Systematic review on bedside electromagnetic-guided, endoscopic, and fluoroscopic placement of nasoenteral feeding tubes. Gastrointest Endosc. 2015;81:836-47.

24. Sethi S, Wadhwa V, Thaker A, Chuttani R, Pleskow DK, Barnett SR, et al. Propofol versus traditional sedative agents for advanced endoscopic procedures: a meta-analysis. Dig Endosc. 2014;26:515-24.

\section{Publisher's Note}

Springer Nature remains neutral with regard to jurisdictional claims in published maps and institutional affiliations.

\section{Ready to submit your research? Choose BMC and benefit from}

- fast, convenient online submission

- thorough peer review by experienced researchers in your field

- rapid publication on acceptance

- support for research data, including large and complex data types

- gold Open Access which fosters wider collaboration and increased citations

- maximum visibility for your research: over $100 \mathrm{M}$ website views per year

At BMC, research is always in progress.

Learn more biomedcentral.com/submissions 